\title{
Developing Mobile Collaborative Learning Applications for Mobile Users
}

\author{
http://dx.doi.org/ijim.v5i4.1823 \\ K. B. Lee \\ University of Northern Virginia, Annandale, Virginia, USA
}

\begin{abstract}
With the rapid growth of wireless communication technologies, the number of mobile applications have been developing. One of them is approaching the mobile learning area since it is unique in its own way and offers learning opportunities anywhere and anytime. Mobile collaborative learning (MCL) is a small group learning application, and it is a new research area in which students can obtain knowledge about a topic and concept via communicating with other students by mobile devices. This paper introduces the theoretical and technical foundations for designing and developing an effective MCL environment. Also, the paper describes a new approach for building the MCL application towards the latest mobile technology. The prototype will be constructed using the Android operating system with suggesting necessary infrastructure and middleware. Finally, the paper include the result of a usability test to find valuable hidden facilitating issues to efficiently access and obtain the class contents in a collaborative learning environment.
\end{abstract}

Index Terms-Mobile Learning, Mobile Collaborative Learning, Usability Test, Usability Factors

\section{INTRODUCTION}

In the last few years, mobile devices have grown in popularity and their technologies have become pervasive, ubiquitous and networked with enhanced capabilities for rich social interactions. Also, many different types of technologies have been adding to the popularity of the mobile devices changing the educational landscape and programs of colleges and universities. Mobile learning is a new research area. The several attractive mobile learning tools have been designing and developing by integrating with the emerging technologies.

With the importance of collaborative learning as a pedagogical method in personal learning and collaborative experience, the learning application has been implanted from elementary schools to universities. Mobile technology has been adapted as a main communication since it is well suited in engaging collaborative learning environments. The concept of mobile collaborative learning (MCL) is completely different from classroombased learning. This type of pedagogical learning method provides many possibilities, such as providing opportunities to groups of people working in same or different organizations to accomplish a specific goal using mobile devices.

Therefore, the demand of collaborative learning over the mobile device has been increasing as a major education element. Major research challenges are raised in developing MCL for educational object such as sharing knowledge, requesting for modified contents, fully accessing to enterprise data warehouse (EDW), delivering large rich multimedia contents, selecting technological components in designing appropriate architecture and adapting application protocols. In this paper, I proposed a conceptual prototype including a communication protocol to deliver rich multimedia contents, improve knowledge sharing process, provide easy to access and contain useful content modification facilities. Another contribution in the paper is to indicate usability issues which are necessary considered to design the application to obtain succinct information and fast feedback through the MCL.

There are various classifications of mobile learning systems in the previous literature. Here, I discuss the salient features of related published articles which contributed to the work. X. Su. and others [1] proposed a four layer framework prototype for multimedia content generation in mobile collaborative systems. The proposed framework provides the support for users, devices and session management skill. This research offers a novel framework for multimedia content generation, representation and delivery for mobile collaboration; however, the framework is not completely elaborated and lacks incorporation of components. V. Zanev and R. Clark [2] suggested the procedures for developing a prototype used for wireless course management system focus on login and authentication, wireless syllabus, wireless calendar and wireless testing. F. Lahner and H. Nosekabel [3] implemented a program which can support e-learning contents to be displayed on computers, and the system structure provides users with the facility to obtain same contents via mobiles. J. Barbosa and others [4] proposed a prototype for undergraduate course reference, called Grefe, which employs mobile and ubiquitous computing. Authors claimed their approach will improve academic and learning activities. The prototype is based on user profile which stores the information regarding learning process and uses location system, so it can identify the user's physical location and support learning procedures by generic architecture; however, the proposed prototype does not provide mobile collaborative learning. A. Druin and others [5] discussed the prototype for their ongoing participatory design project with intergenerational design group to create mobile application and integrate into ip Phone and ipod touch platforms. C. Bouras and others [6] introduced INVITE architecture and discussed the user requirements to meet the demand of e-learning in collaborative virtual environment. It can show the necessary technology and standards for designing MCL.

\section{MOBILE LEARNING ENVIRONMENT}

The mobile learning system should include greater access to necessary content and information timely, reduced cognitive load during learning tasks and increased interaction with users and other systems. Also, the 
learning application plays as a default media player supporting audio and video files, and opens the lecture materials without discontinuity. Basically, in the mobile learning environment, the following running options must be included:
- $\quad$ Lecture Video
- Lecture Note
- Audio
- Quiz and Test
- Assignment
- Discussion
- Grade

To shape a culturally sensitive learning experience, mobile devices will require multimedia oriented network environments. Thus, it is important for designing and developing the wireless based middleware architecture supporting mobile learning system requirements.

\section{A. Requirenments for the System}

In the mobile learning environment, the hardware systems are classified by the type of mobile devices (notebooks, Tablet PCs, PDAs, cell phones, or smart phones) and the type of wireless communication technologies (GSM, CDMA, WiFi, IEEE 802.11, Bluetooth, etc.) according to the information and communication technologies (ICT).

\section{B. Proposed Infrastructure}

The mobile learning system should be designed to support the mobile learning application using a wide variety of languages, platforms and technologies. The learning system should be designed on the client-server based infrastructure to avoid running overload on mobile devices. In the client side, mobile devices, notebooks, Tablet PCs, PDAs, cell phones, smart phones and other devices can access to the content server using wireless technologies. M. Shanmugapriya and A. Tamilarashi [9] suggested the mobile learning architecture in their previous paper as seen in the Fig. 1.

\section{Possible Middleware for Mobile Learning}

In the mobile learning system, the middleware structure must be a framework supporting students' learning work by using computers and communication vendors in a geographically distributed ubiquitous environment. Many different types of media and streaming files will be running for learning methodologies to deal with illiteracy, education programs and other services. To implement a system, it will require a user-oriented design approach to correctly support educational goal and know usability issues, and also a proper wireless communication protocol fitting for learning mode in mobiles. For constructing these middleware, the university should need to contact a mobile service provider which is able to support elearning. This approach can be more helpful for the institution to provide online course preparations on the interest of students.

\section{Learning Applications}

The mobile learning application is a software product for mobile devices. To access the lectures on an individual student mobile device, the required application should be designed to be downloaded from their university website or other places. This type of software release would be more secure and comfortable for students. Once completing the download, it must be installed into their mobile devices. After installation, the application displays a process icon on the mobile screen indicating the group members who are currently accessing the topic or class. The initial display should contain the titles and abstraction of the courses that a student has registered. Basically, to be a successful learning application, the learning tool includes collaboration processes, discussion topics, class lectures, tests, interactive quizzes and so on.

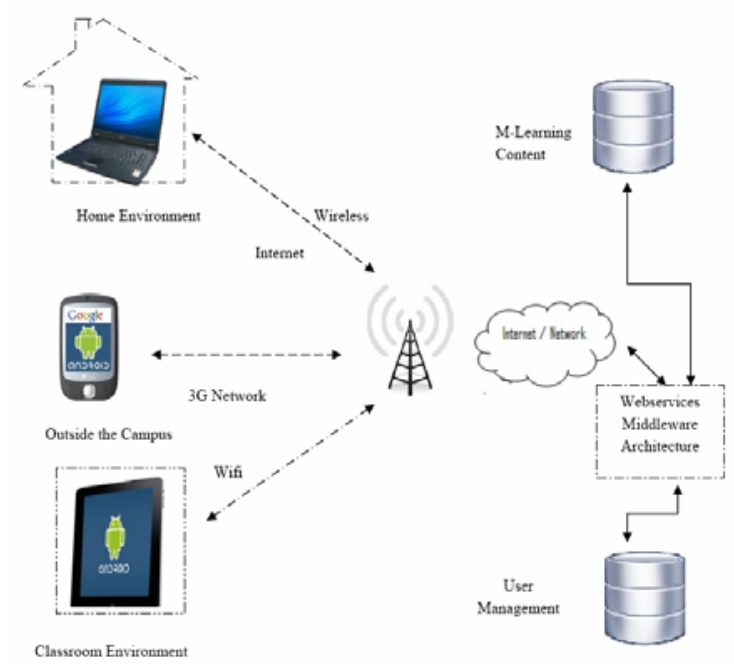

Figure 1. Mobile learning applications for ubiquitous learning environment ${ }^{1}$

\section{E. Other Consideation Factors}

Mostly, the mobile learning materials can be obtained from various digital content service providers. The learning materials should be placed on a server where data or files can be easily saved or retrieved upon users' request. Therefore, many factors will be considered for designing and developing, EDW, wireless protocols and software applications to be able to communicate with students without errors. Furthermore, we should consider that a mobile device by itself includes several weak points in hardware, such as low CPU usage, small memory size, short battery life and others, and also it can make many other security related issues to be used in communication and network.

\section{CONCEPTUAL MidDlEWARE ARCHITECTURE}

To make a successful collaboration meeting the user expectations, I needed to organize the architecture with the support of the latest technologies. Various conceptual collaborative architectures have been proposed so for. The learning system for this prototype adapted and modified four-layered components of collaborative framework proposed by X. Su and others [1], which consists of content generation layer, communication layer, content regeneration layer and content visualization layer. The architecture has been optimized and clearly defined with inclusion of new sub components, and each layer has been assigned a different responsibility. Fig. 2 depicts the conceptual and architectural framework for the mobile collaborative learning environment.

${ }^{1}$ M. Shanmugapriya, and A. Tamilarasi, "Designing an M-Learning Application for a Ubiquitous Learning Environment in the Android Based Mobile Devices Using Web Services," Indian Journal of Computer Science and Engineering (IJCSE), 2011. 


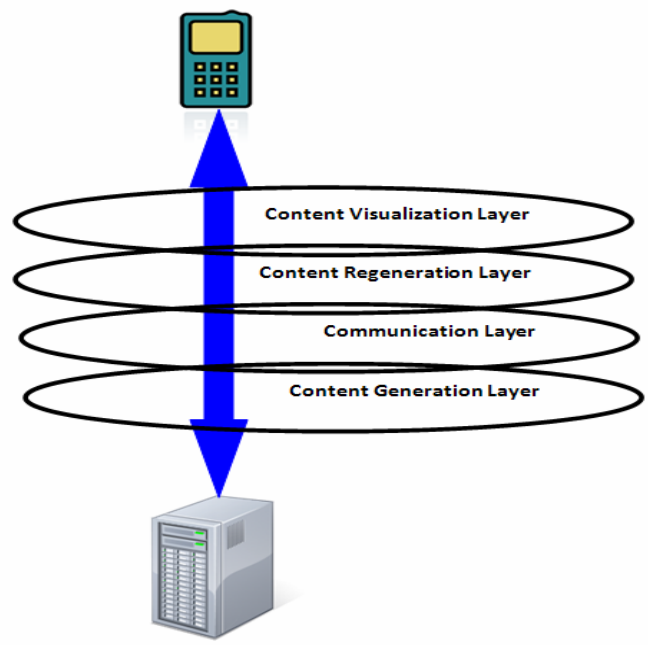

Figure 2. Mobile learning applications for ubiquitous learning environment

\section{A. Content Generation Layer}

The content generation layer is the main component of collaborative framework. If clients require contents, then they send the request message to the content server for delivering required contents. The request message includes device profile, status of previous network condition and requested URL.

\section{B. Communication Layer}

This layer functions as a transport layer. By detecting network status and amount of data decides whether to store messages and to use additional function such as content fragmentation. In a case of no network connectivity, forward the stored messages to the recipient.

\section{Content Regeneration Layer}

This layer performs two types of tasks: first, it forwards the content to display and visualization layer for next process; second, if clients request to modify the content in the middle of process, then also starts to work on the demand.

\section{Content Disaply and Visualization Layer}

The function of this layer is to display and hand over the contents to clients. The layer can obtain the contents from several media manager, and then start to display the contents in several types of forms, graphs, images, voice and others as per the client request. Therefore, the main function of this layer is to translate the source program into an object program that is done with the support of parse engine.

\section{PROPOSED MCL ENVIRONMENT}

Mobile collaborative learning (MCL) is a revolution for education, which allows users to obtain computer-based information through mobile devices. Using MCL provides various advantages, such as context awareness, portability, connectivity and social interaction. As I mentioned early, a mobile can be a successful tool for the collaboration allowing students to share the information to achieve pedagogical activities.

The prototype for MCL is designed for students to easily make collaborations with a mobile anywhere and anytime. Generally, the prototype requires enterprise database warehouse (EDW) to help students find and search necessary information or contents. When logging into a class, initially EDW provides standard course information which comprises of textbook information, course name, course ID, course description, discussion topic and others. Fig. 3 is showing the MCL running processes on the prototype.

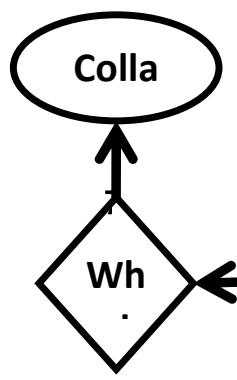
pe.

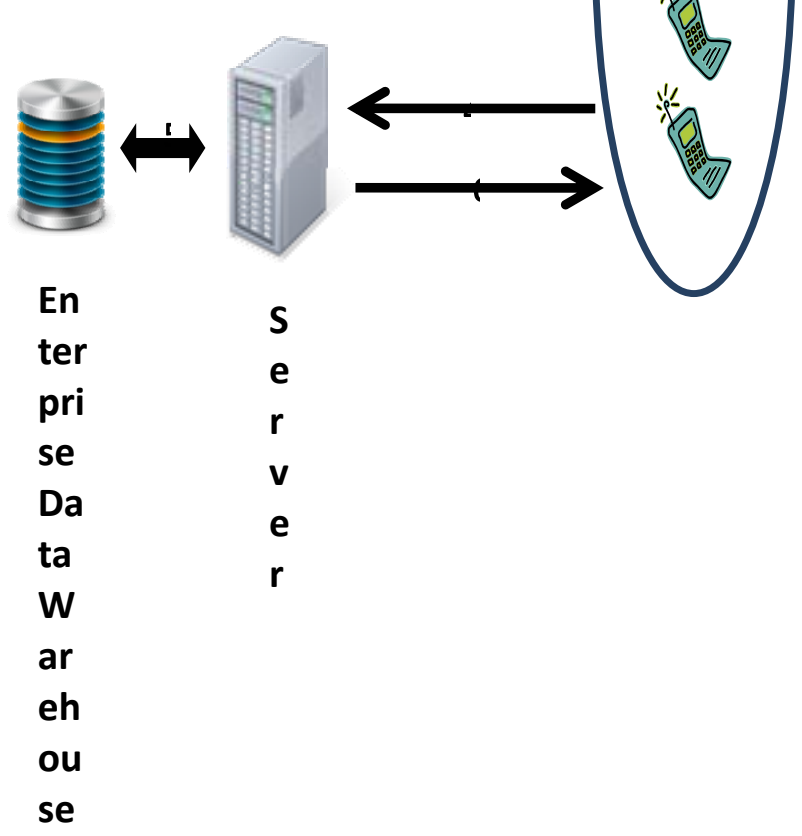

Figure 3. Process for obtaining the course contents through MCL

To be in a well-organized collaborative learning environment, it is very important to set suitable group members and build a study forum. Communication with the forum is necessary to construct a more get-together and affinity group, and also it accommodates easy to set a goal and find the same goal. Generally, the following activities will be required for the MCL application [10]:

- Grouping members

- Monitoring each member

- Displaying member status

- Synchronizing multiple discussion

- Delivering messages 

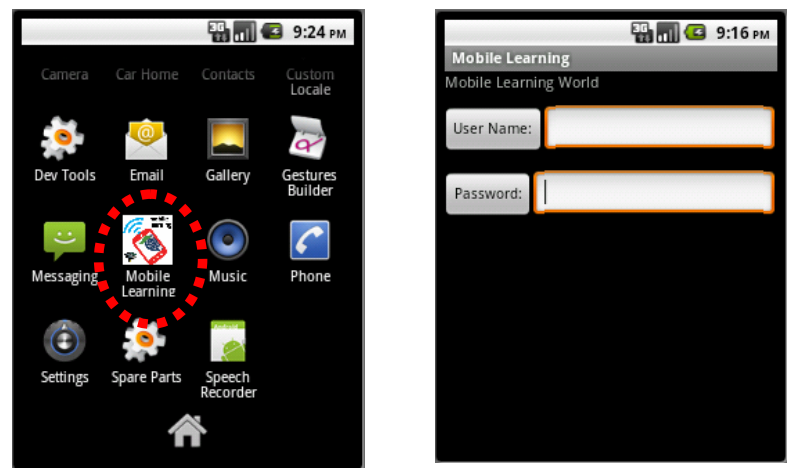

Figure 4. Running an MCL application on mobile devices

\section{A. Authentication Process}

Downloaded application should be installed on the devices displaying an icon as seen in Fig. 4. To protect user profile and system security, the MCL requires authentication process. Thus, after executing the application, it should request for user authentication in order to avoid fault users or loosing priority data as seen in Fig. 4.

Once the process has completed, the application should forward to a new page containing the course that a student has registered for the quarter or semester.

\section{B. Callaborative Learning Process}

After the completion of the login process, a new application "group" with new features is ready to support a user to mange a group communication method as seen in Fig. 5. The Android mobile operating system consists of several software stacks, so by incorporating net news wire software with RSS 2.0 is able to support an MCL application by insulting the following supplementary instructions:

- Add a new application called "group" in the application section by using Java programming.

- Extend resource manager and activity manager to provide extra responsibilities for controlling different features of "group" application.

- Modify libraries section easy to construct data storage.

- Display Linux kernel drivers to support MCL activities.

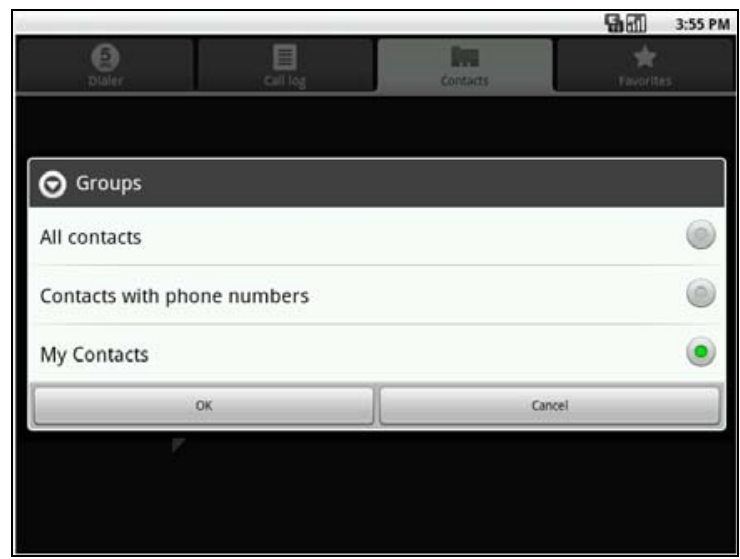

Figure 5. Running a "group" application on Android OS

\section{Running an MCL Prototype}

For more improving a collaborative learning application, systematic communication among of group members is an essential factor. Sending and receiving messages among of group members and forwarding/distributing/sharing files within members are key functions in an MCL application. Students can be asked to create and send a message within a selected group if necessary.

The MCL application prototype, "group" is shown with the Android operating system consisting of control option and delivery option in Fig. 6. Based on two options on the prototype, students are able to set initial learning environment. The control option performs the functionalities adding, deleting, and editing contact, and is able to contact the current group and make a new group if necessary. The delivery option functions to set executing methods for receiving and sending information or messages. In the given option, students can receive and save files from a server when requesting contents. This type of menu structure provides much flexibility for the students to archive their preference.
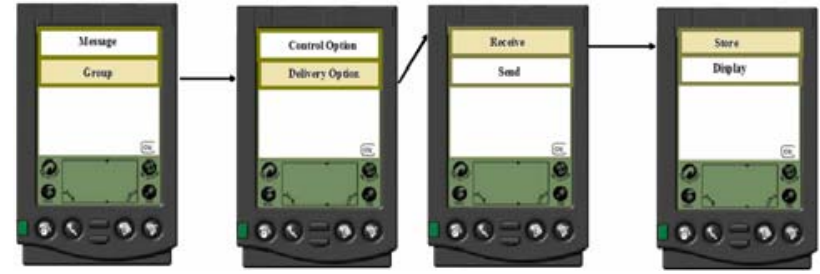

Figure 6. Receiving contents from a server in client's mobile

Accordingly, additional setting options are provided on the prototype. Students will be able to adapt media interface to see, read, listen and watch files, so they can manipulate the different types of materials according to the file format. In any case, the application includes an embedded media player to retrieve the lecture files and display other related materials.

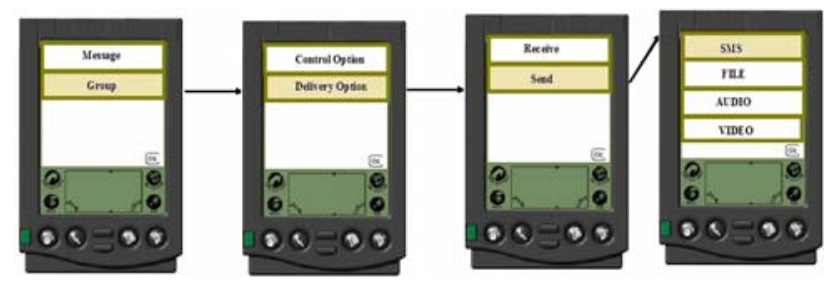

Figure 7. Requesting a client to participate in MCL

When the students finish an initial setting process for manipulating data or materials in its menu and RSS feed, then the setting information will be notified to the collaborating group members through multicasting messages. The process for sending the multicasting message is shown in Fig. 7.

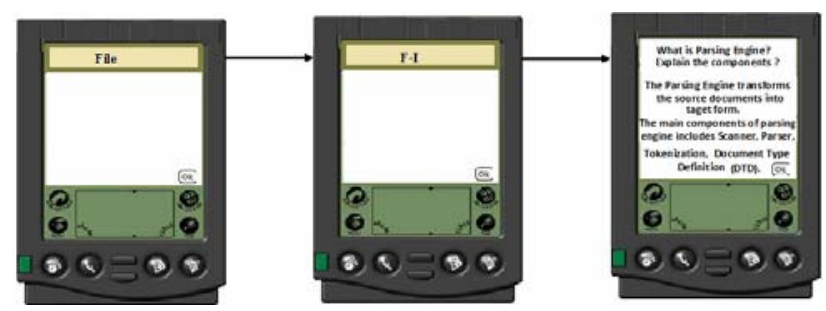

Figure 8. Sharing a file in MCL 


\section{Developing Mobile Collaborative LeARning ApPlications for Mobile Users}

After all collaborating members have completely recognized file processing conditions, then students required for collaboration can start to download the data from the RSS feed, and they can start to share all materials during MCL running time as seen in Fig. 8. Depending on a user request, a simple video file can be delivered for giving more knowledge about the topic or content. Fig. 9 depicts the running video file on the MCL application.

In addition, students can install group option to manage group condition with their individual options. By manipulating collaborating group options in the prototype, a student is able to start a new collaboration process, and can add/join to other group to find information and obtain knowledge as seen in Fig. 10.

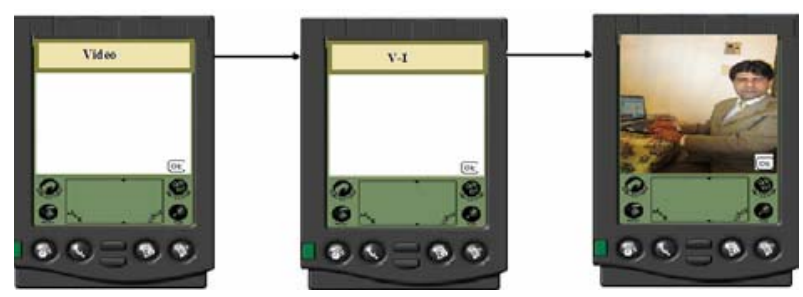

Figure 9. Sharing a video for MCL

With the integration of "group" application, this type of an MCL application in mobile devices can accommodate collaborating members to easily share their resources and discuss a topic or content with group members.

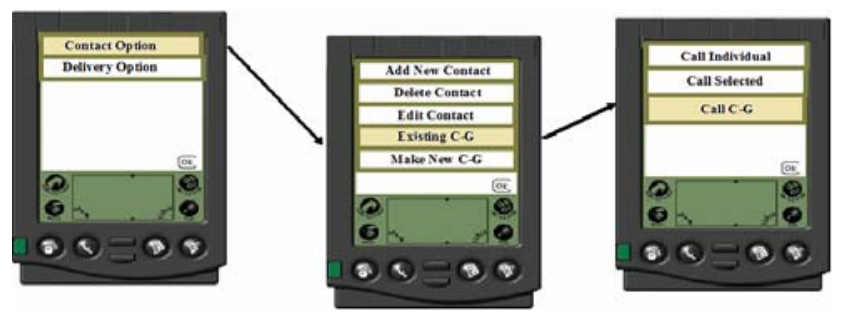

Figure 10. Collaboration for achieving the objectives

\section{FINDING UsABILITY FACTORS}

One of the highly challenging tasks for designing and developing the prototype is to understand what user requirements are and how to reflect and satisfy the required functionalities of application and architecture. Thus, conducting usability test is a necessary process to find basic user needs and satisfy the user requirements. To develop the testing procedure, we will consider the combined testing approach based on heuristic and other formal methods [11].

\section{A. Collecting Users}

To find meaningful issues, 106 testing users who are 58 students, 23 teachers, 14 teaching assistants and 11 administrators from fifteen universities are involved. As to finding pilot usability issues, these people would be joined in the test as online status.

\section{B. Selecting Evaluation Items}

In order to take initial valuable suggestions for building an efficient MCL structure, I have surveyed evaluation items via the previous literature [7][8], then identified and collected the necessary items in Table I. All of the items were selected based on the pedagogical needs for supporting MCL throughout interviews and consultation with experts. To classify and find more valuable usability items, a heuristic based questionnaire test will be conducted by the above users using the evaluation items.

TABLE I.

EVALUATION ITEMS

\section{Selected Evaluation Items}

- $\quad$ Should be easy to use

- Asynchronous collaboration

- Synchronous collaboration

- $\quad$ To support multi model MCL

- $\quad$ To provide archive updating

- $\quad$ Should be user friendly interface

- $\quad$ To get a help from middle ware

- To give virtual support

- To provide application sharing facility and make easy text communication

- To provide admission functionality, if any user wants to participate in middle of the session

- The administrators should be provided the opportunities to record the collaborative activities of students and teachers during the whole session or any specific period of time

- To provide the opportunities for interactive and shard white board

- $\quad$ Users may need short start time for collaboration

- $\quad$ Server should provide content adoption service

- The students should have alternative choices in selecting any topic for discussion

- The students should have access to check the comments given by teacher regarding their performance and grades

- The teacher should include critical notes for the performance of each student after completion of MCL session and provide the feedback to improve in future

- $\quad$ To provide audio and video communication only

- To provide connectivity management support

- To provide the support for session management

- To provide the checking facilities to instructor to check the group members

- To provide the freedom of thoughts to participating group members

- $\quad$ Server should give the message of information updating

- To provide the facility of translation of audio, video and text to other languages

- Client should give notification of his/ her availability

- To provide the support for user role

- Portfolio should be created in order to store an information regarding the course

- $\quad$ To include group manager component

- The methods of communication should be direct or mediated

- The available digital materials should be integrated easily

- Instructor should dedicate time to monitor the progress of participating members

- To provide the support to handle the shared information

- To provide privacy and safety

- To provide the facility to contact and invite the participating for collaboration

- The communication should be based on broadcast with support of multicasting

- To make small participating group for collaboration

- To provide support for floor control administration

- To be flexible to collect and extract the data

- To provide text, graphs, images, audio and video services to meet the requirements of related course of study

- The teachers should have complete access to administer their courses and evaluate the progress of students 


\section{Conducting the Test for Finding Usability Factors}

The usability test is completed by 106 people using Vista survey. Basically, all of questions focus on finding parameters which are mostly efficient factors when designing the MCL application. To completely evaluate each item, five-point Likert scale method is employed. In the testing questionnaires, the five-point Likert items are specified Strongly Agree $=1$, Agree $=2$, Neutral/No Opinion $=3$, Disagree $=4$ and Strongly Disagree $=5$. Finally, I sort out main points from the obtained result, and add mean value to each item, basic requirement $=$ (strongly agree + agree + no opinions) $/ 3 \times 100$. The final result can be shown in Table II.

According to the results, we can set a new plan to modify and redesign the prototype, and then conduct the final empirical testing over the new MCL application. All these testing procedures shall be useful to understand the type of applications required for designing and developing the new MCL application.

TABLE II.

MEAN VALUE FOR SELECTED FACTORS

\begin{tabular}{|c|l|c|}
\hline No & \multicolumn{1}{|c|}{ Description of Basic Requirement } & $\begin{array}{c}\text { Mean } \\
\text { Response }\end{array}$ \\
\hline 1 & Should be easy to use & $100 \%$ \\
\hline 2 & Should be User friendly interface & $100 \%$ \\
\hline 3 & To be flexible to collect and extract the data. & $99.69 \%$ \\
\hline 4 & $\begin{array}{l}\text { To provide text, graphs, images, audio and } \\
\text { video services to meet the requirements of } \\
\text { related course of study }\end{array}$ & $99.49 \%$ \\
\hline 5 & $\begin{array}{l}\text { The teachers should have complete access to } \\
\text { administer their courses and evaluate the } \\
\text { progress of students }\end{array}$ & $98.99 \%$ \\
\hline 6 & $\begin{array}{l}\text { To support multi model MCL } \\
\text { opportunities to record the collaborative } \\
\text { activities of students and teachers during the } \\
\text { whole session or any specific period of time. }\end{array}$ & $98.09 \%$ \\
\hline 8 & $\begin{array}{l}\text { The teacher should include critical notes for } \\
\text { the performance of each student after }\end{array}$ & $97.48 \%$ \\
\hline 9 & $\begin{array}{l}\text { The Students should have alternative choices } \\
\text { in selecting any topic for discussion. }\end{array}$ & $97.28 \%$ \\
\hline 10 & $\begin{array}{l}\text { The Students should have access to check the } \\
\text { comments given by teacher regarding their } \\
\text { performance and grades }\end{array}$ & $97.28 \%$ \\
\hline 11 & $\begin{array}{l}\text { To provide privacy and safety } \\
\text { participating for collaboration }\end{array}$ & $88.35 \%$ \\
\hline 12 & $\begin{array}{l}\text { To provide the facility to contact and invite the } \\
\text { padministrators should be provided the }\end{array}$ & $83.83 \%$ \\
\hline
\end{tabular}

\section{CONCLUSION AND FUTURE WORKS}

The main objective of designing and developing the conceptual-based MCL architecture is to obtain the learning materials on hand-held devices particularly on mobile devices. When applying the MCL system, students should have many learning advantages, such as accessing the course contents anywhere and anytime. Throughout the research paper, several contributions can be pointed out. First, I have adapted four-layered architecture which was explained previously. The adapted four-layer architecture can provide an efficient and fast way of delivering the contents to mobile devices. Second, I have introduced the client-server based an MCL prototype architecture for improving education environments. The design and development of MCL using client-server based architecture should be the fast and secure method to share and deliver the contents. Third, I have proposed and implemented the MCL application prototype with recommending and explaining valuable suggestions on how to use the Android operating systems and how to meet the course objective. Finally, I have discussed the MCL user requirements by conducting a usability test to design and develop a new MCL application to meet the pedagogical requirements of students, teachers, teacher assistants and administrators.

Although, this approach is to devise a basic method for efficiently building an MCL application, there are still uncovered problems applying for a commercial mobile application directly. However, the architecture will properly meet the current challenge of MCL and I will keep focusing on implementing the whole group application to meet new user needs and requirements. I look forward to continuing my research and developing the application based on the future progress of mobile software and hardware performance. Therefore, the biggest contribution of the paper is to provide a small step for how to design and implement MCL in order to support the pedagogical needs.

\section{REFERENCES}

[1] X. Su, B. S. Prabhu, C. Chu, R. Gadh, "Midleware for multimedia mobile collaborative system," in IEEE $3^{\text {rd }}$ Annual Wireless Telecommunications Symposium, May 14-15, 2004, California, USA.

[2] V. Zanev and R. Clark, "Wireless Course Management System," 43rd ACM Southeast Conference, March 18-20, 2005, Kennesaw, GA, USA.

[3] F. Lahner and H. Nosekabel, "The Role of Mobile Devices in ELearning First Experiences with a Wireless E-Learning Environment," Proceedings of the IEEE International Workshop on Wireless and Mobile Technologies in Education, August 29-30, 2002, Vaxjo, Sweden

[4] J. Barbosa, R. Hahn, Debora N. F. Barbosa, Claudio, Claudio, F. R. Geyer, "Mobile and Ubiquitous Computing in an Innovative Undergraduate Course,” SIGCSE '07, March 7-10, 2007, Covington, Kentucky, USA.

[5] A. Druin, B. B. Benderson, A. Quinn, "Designing Inter generational Mobile Storytelling,” IDC 2009-Workshops, June 3-5, 2009, Como, Italy.

[6] C. Bouras, G. Hornig, V. Triantafillou, T. Tsiatsos, “Architectures Supporting e-Learning through Collaborative Virtual Environments," The Case of INVITE, ICALT 2001, pp. 13-16. Madison, WI, USA.

[7] V. Hinze-Hoare, Designing a Collaborative Research Environment for Student and their Supervisors (CRESS), School of Electronics and Computer Science, University of Southampton, UK, 2007.

[8] S. Ochoa, R. Alarcon, L. Guerrero, "Understanding the Relationship between Requirements and Context Elements in Mobile Collaboration," HCII 2009, LNCS 5612, pp. 67-76, Springer, Heidelberg, 2009.

[9] M. Shanmugapriya, and A. Tamilarasi, “Designing an M-Learning Application for a Ubiquitous Learning Environment in the Android Based Mobile Devices Using Web Services," Indian Journal of Computer Science and Engineering (IJCSE), 2011.

[10] C. Wang, "The Development of Collaborative Learning Environment with Learning Blogs.” Journal of Software, Vol. 4. No. 2, April, 2009.

[11] K. B. Lee and R. A. Grice, "Developing a New Usability Testing Method on Mobile Devices,” IPCC 2004, IEEE Professional Communication Society, Minneapolis, 2004, MN, USA.

[12] K. B. Lee and A. Razaque, "Suggested Collaborative Learning Conceptual Architecture and Applications for Mobile Devices.” HCI International 2011, July 9-14, 2011, Orland, Florida, USA. 


\section{SHORT PAPER}

\section{DeVeloping Mobile Collaborative LEARNing APPLiCATIONS FOR MOBILE USERS}

\section{AUTHOR}

K. B. Lee is an assistant professor of the University of Northern Virginia in Annandale, VA. He was a senior engineer, Mobile Communication Division, SAMSUNG Electronics Co., Suwon, KOREA. He received his PhD in
Engineering Science from Rensselaer Polytechnic Institute in Troy, NY, USA. He is a member of ACM.

Received 25 September 2011. Published as resubmitted by the author 27 September 2011. 